\title{
PERCEPÇÃO DA IMAGEM CORPORAL EM ÁRBITROS DE FUTEBOL
}

\author{
Alberto Inácio da Silva ${ }^{1}$ \\ Diego Augusto Santos Silva \\ Mauro Ricetti Paes ${ }^{3}$
}

SILVA, A. I. da; SILVA, D. A. S.; PAES, M. R. Percepção da imagem corporal em árbitros de futebol. Arq. Cienc. Saúde UNIPAR, Umuarama, v. 22, n. 3, p. 187-191, set./dez. 2018.

\begin{abstract}
RESUMO: O objetivo deste trabalho foi analisar a percepção da imagem corporal e identificar fatores individuais associados a esta percepção em árbitros de futebol do Brasil. Participaram do estudo 94 árbitros profissionais pertencentes à Federação Catarinense de Futebol, sendo todos do sexo masculino. Para a identificação da percepção da imagem corporal os árbitros responderam o Body Shape Questionnaire. A massa corporal e a estatura autorrelatadas foram utilizadas para o cálculo do índice de massa corporal. Observou-se que $64,9 \%$ dos árbitros estão insatisfeitos com a imagem corporal, sendo que o índice de massa corporal médio foi de $24,4 \mathrm{~kg} / \mathrm{m}^{2}$. Quanto ao tempo de treinamento, observou que os árbitros treinam em média 3 dias/semana, cerca de 60 minutos/treino. Conclui-se que a maior parte dos árbitros está insatisfeita com a imagem corporal, sendo que, aqueles que passam mais tempo em treinamento estão mais satisfeitos com a imagem corporal. PALAVRAS-CHAVE: Árbitro. Futebol. Imagem corporal
\end{abstract}

\section{BODY IMAGE PERCEPTION IN SOCCER REFEREES}

\begin{abstract}
The purpose of this study was to analyze the body image perception and identify individual factors associated with this perception in Brazilian soccer referees. The study included 94 male professional referees registered at the Santa Catarina Soccer Federation. For the identification of body image perception, referees answered the Body Shape Questionnaire. Self-reported data on body weight and height were used to calculate body mass index. It was observed that $64.9 \%$ of referees were unsatisfied with their body image, and the mean body mass index was $24.4 \mathrm{~kg} / \mathrm{m}^{2}$. Regarding time spent in training, it could be observed that the referees train on average 3 days/week, for approximately 60 minutes/workout session. It can be concluded that most referees were unsatisfied with their body image, while those who spent more time in training are more satisfied with their body image.
\end{abstract}

KEYWORDS: Body image. Referee. Soccer.

\section{Introdução}

A crescente popularização do futebol influenciada pela maior visibilidade dos meios de comunicação contemporâneos (televisão, internet e smartphones), dos valores comercias e financeiros atrelados a eventos como a Copa do Mundo e campeonatos continentais, tem colocado o futebol como um dos mais importantes espetáculos do esporte no mundo. Nesse contexto, a valorização dos elementos (atletas, árbitros, jornalistas) ligados diretamente ao jogo também tem se popularizado. Entre estes elementos destaca-se o árbitro de futebol, que tem como função julgar as ações relacionadas ao jogo (LAGO-PEÑAS; GÓMEZ-LÓPEZ, 2016).

Devido ao grande volume de ações motoras e cognitivas desenvolvidas pelos árbitros durante a partida começaram a surgir trabalhos científicos descrevendo o perfil morfológico do árbitro. Um dos pioneiros em publicar trabalhos sobre a composição corporal do árbitro de futebol foram Rontoyannis et al. (1998), que envolveu árbitros gregos em sua pesquisa. No Brasil, o perfil antropométrico do árbitro da Confederação Brasileira de Futebol (CBF), ou seja, dos árbitros e assistentes de elite do Brasil, também foi estudado (DA SILVA; RODRIGUEZ-AÑEZ, 2003).

A discussão sobre o somatotipo do árbitro teve início em 2008 e foi discutida pela primeira vez por Da Silva e Rech, (2008). Neste trabalho os autores apresentaram o somatotipo de árbitros de elite do Brasil. No final de 2008, no âmbito internacional, em um trabalho sobre o somatotipo de árbitros internacionais, foram descritas as características antropométricas do árbitro chileno (FERNÁNDEZ; DA
SILVA; ARRUDA, 2008). Estes estudos, apontaram que os árbitros se encontram com o percentual de gordura superior aos dos jogadores desta modalidade esportiva, não sendo encontrado na literatura nenhum estudo desenvolvido com os árbitros sobre a concepção que este tem em relação a autoimagem corporal.

A imagem corporal pode ser entendida como uma representação multidimensional que configura as representações internas da estrutura corporal e da aparência física que a pessoa tem de si mesmo ou de outrem (TYLKA; WOOD-BARCALOW, 2015). Esta imagem corporal é constituída por quatro dimensões: cognitiva, afetiva, comportamental e perceptiva (CASH; PRUZINSKY, 2002). Dentre estas, a dimensão perceptiva tem sido frequentemente mais utilizada, na área da saúde, na tentativa de avaliar como o indivíduo percebe a forma física (WILLIAMSON et al., 2000). A insatisfação com a imagem corporal pode levar o indivíduo a desenvolver transtorno de imagem (TYLKA; WOOD-BARCALOW, 2015). Um destes transtornos é conhecido como Transtorno Dismórfico Corporal (Body Dysmorphic Disorder), que é caracterizado por preocupação exacerbada com uma irregularidade imaginária na aparência corporal ou pela supervalorização de uma deformação mínima. Este tipo de transtorno é descrito como o mais comum entre pacientes que buscam a intervenções cirúrgicas como solução estética (PHILLIPOU; CASTLE, 2015).

Desta forma, a busca de um corpo perfeito, está levando as pessoas a se submeterem cada vez mais à cirurgias estéticas, que juntas com outras estratégias de modelagem corporal, como a prática de atividades físicas, as dietas restri-

DOI: 10.25110 /arqsaude.v22i3.2018.6574

${ }^{1}$ Docente do departamento de Educação Física da Universidade Estadual de Ponta Grossa. Grupo de Pesquisa em Árbitro de Futebol GPAF. Rua: Henrique Zuber,76 - Vila Estrela. CEP: 84.050-600 - Ponta Grossa - Paraná. e-mail: albertoinacio@bol.com.br ${ }^{2}$ Docente do departamento de Educação Física da Universidade Federal de Santa Catarina. diegoaugustoss@yahoo.com.br ${ }^{3}$ Programa de Pós-Graduação da Universidade Federal do Paraná. Doutorando em Fisiologia. mauroricetti@gmail.com 
tivas, uso de laxantes, diuréticos e esteroides anabolizantes, buscam melhorar a percepção que a pessoa possui de si mesma (PANAYI, 2015).

Dessa maneira, estudos confirmando que inúmeros árbitros estão com o percentual de gordura corporal acima dos jogadores desta modalidade (DA SILVA, 2011; KAMINAGAKURA et al., 2013; ZORAGHI; KHOSHNAM; SOLHJO, 2014) estariam estes satisfeitos com sua imagem corporal? Além disso, como o árbitro de futebol está inserido na sociedade contemporânea que exige a busca de um corpo cada vez mais recomendado pelos meios de comunicação que priorizam a estética (TYLKA; WOOD-BARCALOW, 2015), a investigação dessa temática na população de árbitros de futebol pode servir de auxílio para identificar se esse público tem percepção positiva ou negativa da imagem corporal.

Assim, este estudo tem como objetivo analisar a percepção da imagem corporal e identificar fatores individuais associados a esta percepção em árbitros de futebol do Brasil.

\section{Materiais e Métodos}

Trata-se de um estudo descritivo, com delineamento transversal, realizado com árbitros de Futebol de Campo da Federação Catarinense de Futebol (FCF). A população do estudo foi composta por 120 árbitros de futebol cadastrados junto a FCF. Todos os árbitros foram convidados a participar da coleta de dados. Por fim, participaram do estudo 94 árbitros que aceitaram participar da pesquisa, sendo todos do sexo masculino.

Os dados foram coletados durante a pré-temporada dos árbitros da FCF organizada pelo Sindicato dos árbitros de Futebol de Santa Catarina (SINAFESC) na cidade de Florianópolis (Santa Catarina - Brasil). Foi aplicado um questionário padronizado a fim de selecionar as questões de interesse para a pesquisa. $\mathrm{O}$ estudo atende às recomendações da Resolução 196/96 do CNS e foi aprovado pelo Comitê de Ética em Pesquisa com seres humanos da Universidade Estadual de Ponta Grossa, Protocolo 20357/2011.

A variável dependente do estudo foi a percepção da imagem corporal. Para tanto, empregou-se a escala de silhuetas corporais (STUNKARD; SORENSON; SCHLUSINGER, 1983). O conjunto de silhuetas foi mostrado aos sujeitos, seguido das perguntas: 1) Qual a silhueta que melhor representa sua aparência física atual? Essa questão correspondeu à aparência física real; 2) Qual silhueta você gostaria de ter? Essa questão correspondeu à aparência física considerada ideal pelo respondente. $\mathrm{O}$ grau de insatisfação com a imagem corporal foi estimado pela diferença entre a silhueta real e a ideal. Valores positivos indicam que o sujeito está insatisfeito por excesso de peso e tem o desejo de diminuir a silhueta corporal. Por outro lado, valores negativos indicam insatisfação por magreza e o desejo de aumentar a silhueta corporal. Quando o valor foi igual a zero, isso indicava que o sujeito estava satisfeito com aparência física. Esse instrumento apresenta indicadores satisfatórios de validade e reprodutibilidade para análise do tamanho corporal (KESHTKAR et al., 2010).

As variáveis independentes do estudo foram as características demográficas (idade, tempo de treino na semana, tempo na arbitragem) e o estado nutricional que foram obtidas por meio de auto-relato. Todas as questões referentes às variáveis independentes foram coletadas de forma aberta e posteriormente categorizadas para a análise dos dados. A idade foi categorizada em $\leq 29$ anos e $\geq 30$ anos. Para o tempo de treino semanal questionou-se sobre a frequência semanal de treino e a duração de cada treino, sendo a resposta categorizada em $<300$ minutos por semana ou $\geq 300$ minutos por semana. $\mathrm{O}$ tempo na arbitragem foi categorizado em 0-4 anos, 5-9 anos e $\geq 10$ anos. Para classificação do estado nutricional, a massa corporal $(\mathrm{kg})$ e a estatura $(\mathrm{m})$ foram utilizadas para o cálculo do índice de massa corporal (IMC $\mathrm{kg} / \mathrm{m}^{2}$ ), sendo estas medidas obtidas por meio de auto relato, procedimento validado para adultos brasileiros (COQUEIRO et al., 2009). Adotaram-se os critérios da World Health Organization - WHO (1998), considerando peso normal IMC $\leq 24,9 \mathrm{~kg} / \mathrm{m}^{2}$ e excesso de peso o IMC $\geq 25 \mathrm{~kg} / \mathrm{m}^{2}$.

$\mathrm{Na}$ análise dos dados utilizaram-se os recursos da estatística descritiva (média, desvio padrão e frequências). Para identificar a associação entre a percepção da imagem corporal com as variáveis independentes usou-se o teste Qui-quadrado ou o teste Exato de Fisher. Foi utilizado o programa SPSS versão 10.0, adotando-se um nível de significância de $5 \%$.

\section{Resultados}

Após análise dos dados coletados, observou-se que a média de idade dos árbitros foi de 30,3 anos $(\mathrm{dp}=6,66)$, massa corporal de $77,7 \mathrm{~kg}(\mathrm{dp}=9,8)$, estatura $1,78 \mathrm{~m}(\mathrm{dp}=0,06)$ e índice de massa corporal de $24,4 \mathrm{~kg} / \mathrm{m}^{2}(\mathrm{dp}=2,46)$. Quanto ao tempo de treinamento, observou que os árbitros treinam em média 3 dias/semana $(d p=1,08)$, cerca de 60 minutos/ treino $(\mathrm{d} p=25,8)$ e atuam na arbitragem em média a 6 anos. Observou-se que 64,9\% dos árbitros estão insatisfeitos com a imagem corporal. A tabela 1 apresenta características da amostra do estudo.

Tabela 1: Características descritivas da amostra de árbitros de futebol.

\begin{tabular}{llll}
\hline Variável & Categoria & N & $\%$ \\
\hline Faixa etária & & & \\
& $\leq 29$ anos & 49 & 52,1 \\
& $\geq 30$ anos & 45 & 47,9
\end{tabular}

Status de peso

$\begin{array}{llll}\text { Normal } & 60 & 63,8 \\ & \text { Excesso de peso } & 34 & 36,2\end{array}$

Tempo treino na semana

$\begin{array}{lll}<300 \text { minutos } & 77 & 81,9 \\ \geq 300 \text { minutos } & 17 & 18,1\end{array}$

Tempo de arbitragem

$\begin{array}{lll}0-4 \text { anos } & 41 & 43,6 \\ 5-9 \text { anos } & 33 & 35,1 \\ \geq 10 \text { anos } & 20 & 21,3\end{array}$

Imagem corporal

\begin{tabular}{lll} 
Insatisfeito & 61 & 64,9 \\
Satisfeito & 33 & 35,1 \\
\hline
\end{tabular}


Em relação à insatisfação com a imagem corporal, pode-se observar que a maior parte, $42,6 \%$, está insatisfeita por excesso de peso. Porém, $22,3 \%$ dos árbitros estão insatisfeitos por magreza (figura 1).

Figura 1: Proporção de árbitros de futebol satisfeitos e insatisfeitos com a imagem corporal.

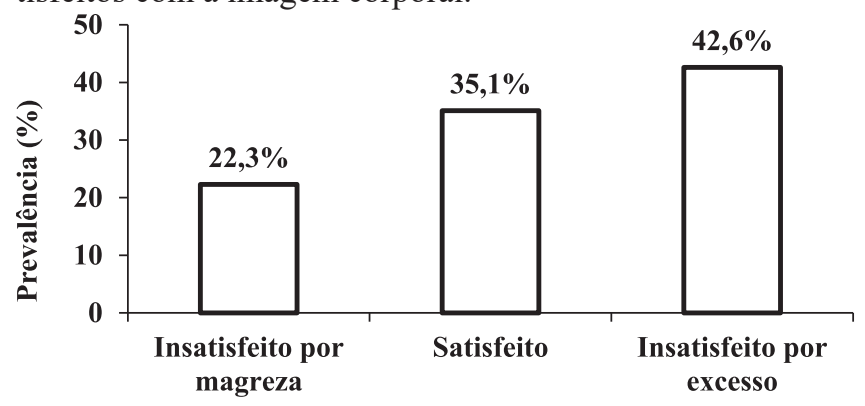

Percepção da Imagem Corporal

A silhueta mais relatada como a percebida como real e a ideal para os árbitros foi a de número 3 e 4, respectivamente. Cinco árbitros declararam que a silhueta real seria a de número 5 e seis árbitros declararam que a silhueta de número 2 seria a ideal (Tabela 2).

Tabela 2: Frequência dos relatos de imagem corporal real, ideal e ideal para o sexo oposto em árbitros de futebol.

\begin{tabular}{ccccc}
\hline \multirow{2}{*}{$\begin{array}{c}\text { Silhueta } \\
\text { corporal }\end{array}$} & \multicolumn{2}{c}{ Imagem real } & \multicolumn{2}{c}{ Imagem ideal } \\
\cline { 2 - 5 } & $\mathbf{N}$ & $\mathbf{\%}$ & $\mathbf{N}$ & $\mathbf{\%}$ \\
\hline 2 & 15 & 16,0 & 6 & 6,4 \\
3 & 26 & 27,7 & 42 & 44,7 \\
4 & 27 & 28,6 & 41 & 43,6 \\
5 & 23 & 24,5 & 5 & 5,3 \\
6 & 3 & 3,2 & - & - \\
\hline
\end{tabular}

Existiu associação entre o tempo de treino semanal e a percepção da imagem corporal, sendo que a maior parte dos árbitros insatisfeitos por excesso de peso $(48,1 \%)$ treinava menos de 300 minutos por semana. Além disso, encontrou-se que os árbitros com menos de cinco anos na profissão estão insatisfeitos por excesso de peso $(51,2 \%)$. Por outro lado, os sujeitos com 10 anos ou mais de arbitragem (45,0\%) estavam mais insatisfeitos por magreza (Tabela 3 ).

Tabela 3: Associação entre insatisfação com a imagem corporal e características pessoais e de treino dos árbitros de futebol.

\begin{tabular}{|c|c|c|c|c|}
\hline \multirow[t]{3}{*}{ Variáveis } & \multicolumn{3}{|c|}{ Percepção da imagem corporal } & \multirow{3}{*}{$p$} \\
\hline & Satisfeito & Insatisfeito por magreza & Insatisfeito por excesso & \\
\hline & n $(\%)$ & n $(\%)$ & n $(\%)$ & \\
\hline \multicolumn{5}{|l|}{ Faixa etária } \\
\hline$\leq 29$ anos & $18(36,7)$ & $08(16,3)$ & $23(47,0)$ & 0,333 \\
\hline$\geq 30$ anos & $15(33,3)$ & $13(28,9)$ & $17(37,8)$ & \\
\hline \multicolumn{5}{|l|}{ Status de peso } \\
\hline Peso normal & $20(33,3)$ & $13(21,7)$ & $27(45,0)$ & 0,812 \\
\hline Excesso de peso & $13(38,2)$ & $08(23,5)$ & $13(38,2)$ & \\
\hline \multicolumn{5}{|c|}{ Tempo treino semanal } \\
\hline$<300$ minutos & $24(31,1)$ & $16(20,8)$ & $37(48,1)$ & $0,033^{*}$ \\
\hline$\geq 300$ minutos & $09(53,0)$ & $05(29,4)$ & $03(17,6)$ & \\
\hline \multicolumn{5}{|c|}{ Tempo de arbitragem } \\
\hline $0-4$ anos & $15(36,6)$ & $05(12,2)$ & $21(51,2)$ & $0,045^{*}$ \\
\hline $5-9$ anos & $11(33,3)$ & $07(21,2)$ & $15(45,5)$ & \\
\hline$\geq 10$ anos & $07(35,0)$ & $09(45,0)$ & $04(20,0)$ & \\
\hline
\end{tabular}
$\mathrm{p}<0,05$.

\section{Discussão}

Este estudo é pioneiro em analisar a percepção da imagem corporal em árbitros de futebol e teve como principal achado que a insatisfação com a imagem corporal se faz presente em sujeitos desta profissão e que a maior insatisfação está relacionada com o excesso de peso. Ademais, o tempo de treinamento semanal e o tempo na arbitragem estiveram associados a esta insatisfação com a imagem corporal, sendo que a maior parte dos árbitros que treinavam menos de 300 minutos por semana e que tinha menos do que cinco anos na arbitragem estava insatisfeita por excesso de peso. Por outro lado, os árbitros há mais tempo na profissão estavam mais insatisfeitos por magreza.

A maior parte dos árbitros $(42,6 \%)$ relatou insatis- fação por excesso de peso, sendo que o excesso de peso foi identificado em 36,2\% da amostra. Esse achado demonstra uma discrepância entre o real status do peso com a percepção da imagem corporal, o que pode indicar futuros transtornos mentais, como a depressão (PIMENTA et al., 2009), distúrbios alimentares como anorexia e bulimia (YEH et al., 2009) e baixa autoestima (ABRAHAM, 2003). Essas condições afetam a qualidade de vida e refletem diretamente o estado de saúde e as relações pessoais do cotidiano. Por esta razão, se faz necessário uma conscientização dos árbitros com intuito de orientá-los para hábitos saudáveis e para manterem bom rendimento físico requerente à profissão.

Neste estudo, os árbitros demonstraram que a silhueta ideal mais relatada foram as de número 3 e 4 . Keshtkar et al. (2010) ao analisar tais silhuetas com o IMC em adultos 
do Iran, relataram que a silhueta 3 corresponde a um IMC em torno de $23 \mathrm{~kg} / \mathrm{m}^{2}$ e a silhueta 4 a um IMC de $26 \mathrm{~kg} / \mathrm{m}^{2}$. Desta forma, acredita-se que os árbitros de futebol analisados no presente estudo entendem que um corpo ideal para a arbitragem poderia ser aquele com IMC entre $23 \mathrm{~kg} / \mathrm{m}^{2}$ e $26 \mathrm{~kg} / \mathrm{m}^{2}$. O componente físico é tido como um dos mais importantes para uma boa preparação do árbitro para assim, efetivar sua atuação no campo de jogo (DA SILVA, 2005). O IMC médio dos árbitros envolvidos neste estudo foi de $24,4 \pm 2,46 \mathrm{~kg} / \mathrm{m}^{2}$. Este valor é semelhante ao valor encontrado em um estudo envolvendo 215 árbitros profissionais, que foi de 24,8 $\pm 2,8$ $\mathrm{kg} / \mathrm{m}^{2}$ (DA SILVA et al., 2011). Helsen e Bultynck (2004) relataram valores de IMC de 24,2 $\pm 2,6 \mathrm{~kg} / \mathrm{m}^{2}$ nos árbitros que atuaram nas finais do Campeonato Europeu de 2000, ou seja, pela média os árbitros estariam com o IMC dentro da normalidade, segundo o valor de referência da WHO (1998), que é de $25 \mathrm{~kg} / \mathrm{m}^{2}$. Entretanto, como comentado anteriormente $36 \%$ os árbitros apresentaram valores de IMC que os classificam como sobrepeso. Fato semelhante foi relatado por Da Silva e Rech (2008) quando estudaram árbitros da CBF e verificaram que o IMC dos árbitros principais em detrimento dos árbitros assistentes era classificado como sobrepeso. Em um estudo envolvendo 188 árbitros gregos, divididos em várias categorias, observou-se que, em todas as categorias, os árbitros foram classificados com sobrepeso, sendo a média desta amostra de 25,9 $\pm 2,1 \mathrm{~kg} / \mathrm{m}^{2}$ (RONTOYANNIS et al., 1998).

Os árbitros catarinenses envolvidos neste estudo afirmaram fazer uma preparação física para arbitrar, em média três vezes por semana, sendo que a duração era em média de 60 minutos. A atividade física praticada pela grande maioria dos árbitros é a corrida aeróbica, sendo ignorado por eles os trabalhos anaeróbicos, ou seja, corridas intermitentes (DA SILVA, 2005). Dados semelhantes foram encontrados por Paes, Fernandez e Da Silva (2011), quando estudaram árbitros paranaenses. Portanto, o que se observa neste estudo é que a grande maioria dos árbitros treina com frequência, duração recomendada para uma pessoa possuir o mínimo de qualidade de vida e não para o aumento das capacidades físicas. Isso também foi observado em outro país, pois Krustrup e Bangsbo (2001), quando desenvolveram um estudo com árbitros dinamarqueses, relataram que o treinamento dos árbitros de alta classe, frequentemente, consistia de corrida aeróbica de intensidade moderada, com percursos entre 3 e $7 \mathrm{~km}$. No presente estudo, foi identificado que os árbitros que treinam menos de 300 minutos por semana, apresentaram maior insatisfação com a imagem corporal por excesso de peso. Este fato pode estar relacionado ao baixo volume de treinamento dos árbitros em comparação aos pares que têm maior volume semanal de treinamento. Um maior volume semanal de treinamento reflete em maior gasto energético e por consequência, em menor quantidade de gordura corporal (WHO, 1998). A literatura reporta que pessoas com maior nível de atividade física tendem a serem menos insatisfeitos por excesso de peso do que as pessoas com baixos níveis de atividade física (KRUGER et al., 2008).

O presente estudo apresenta como limitações: 1) o delineamento transversal, que não permite identificar relações de causalidade entre a percepção da imagem corporal e o tempo de treinamento semanal e o tempo na arbitragem. Além disso, a utilização a escala de silhuetas corporais com desenhos bidimensionais para a avaliação da imagem corpo- ral pode implicar falhas na representação total do corpo e/ou na distribuição da massa de gordura. Contudo, cabe ressaltar que esse trabalho foi o primeiro estudo sobre percepção da imagem corporal em árbitros de futebol e pode servir para reflexões e como informação útil para uma reeducação sobre conscientização corporal destes profissionais.

Pode-se concluir que a insatisfação com a imagem corporal se faz presente em árbitros de futebol, em que a maior insatisfação está relacionada com o excesso de peso. Ademais, a maior parte dos árbitros que treinam menos de 300 minutos por semana estava insatisfeita por excesso de peso. Por outro lado, os árbitros com 10 ou mais anos de profissão estavam mais insatisfeitos por magreza.

\section{Conclusão}

Os resultados desta pesquisa demonstraram que a maioria dos árbitros estão insatisfeitos com sua imagem corporal. Destes, a maior parte está insatisfeito por estar com excesso de peso (42\%). Este excesso de peso sofre influência da forma adotada para a preparação física destes árbitros, pois foi diagnosticado que eles treinam em média três vezes por semana com duração média de 60 minutos. Neste sentido, sugere-se que as Federações e a Confederação Brasileira de Futebol ofereçam aos seus árbitros programas de condicionamento físico associados à orientações nutricionais visando melhorar o perfil atlético, e assim, prolongando a vida útil dos mesmos, evitando que eles deixem de arbitrar por estarem com excesso de peso ou falta de condições físicas, uma vez que as experiências acumuladas pelos árbitros ao longo de sua carreira é um dos fatores determinantes para uma boa arbitragem.

\section{Referências}

ABRAHAM, S. F. Dieting, body weight, body image and self-esteem in young women: doctors' dilemmas. The Medicine Journal of Australia, v. 178, n. 12, p. 607-611, 2003.

CASH, T. F.; PRUZINSKY, T. Body Image: A Handbook of Theory, Research, and Clinical Practice. New York, Guilford Press, 2002.

COQUEIRO, R. S. et al. Medidas auto-referidas são válidas para avaliação do estado nutricional na população brasileira? Revista Brasileira de Cineantropometria \& Desenvolvimento Humano, v. 11, n. 1, p. 113-119, 2009.

Da SILVA, A. I. Bases científicas e metodológicas para o treinamento do árbitro de futebol. Curitiba, Imprensa da UFPR, 2005.

DA SILVA, A. I. Somatotype and physical fitness of the assistant referees in soccer. International Journal of Morphology, v. 29, n. 3, p. 792-798, 2011.

DA SILVA, A. I. et al. Somatotype and body composition of Brazilian football (soccer) referees. Archivos de Medicina del Deporte, v. 28, n. 144, p. 238-246, 2011.

DA SILVA, A. I.; RECH, C. R. Somatotipo e composição 
corporal de árbitros e árbitros assistentes da CBF. Revista Brasileira de Cineantropometria \& Desenvolvimento Humano, v. 10, n. 2, p. 143-146, 2008.

DA SILVA, A. I.; RODRIGUEZ-AÑEZ, C. R. Níveis de aptidão física e perfil antropométrico dos árbitros de elite do Paraná credenciados pela Confederação Brasileira de Futebol (CBF). Revista Portuguesa de Ciências do Desporto, v. 3, n. 3, p. 18-26, 2003.

FERNÁNDEZ, V. G. E.; DA SILVA, A. I.; ARRUDA, M. Perfil antropométrico y aptitud física de árbitros del fútbol profesional chileno. International Journal of Morphology, v. 26, n. 4, p. 897-904, 2008.

HELSEN, W.; BULTYNCK, J. B. Physical and perceptualcognitive demands of top-class refereeing in association football. Journal of Sports Science, v. 22, n. 2, p. 179-189, 2004

KAMINAGAKURA, E. I. et al. Análise comparativa do perfil antropométrico de árbitros e jogadores de futebol. Arq. Ciênc. Saúde UNIPAR, v. 17, n. 1, p. 23-30, jan./abr. 2013

KESHTKAR, A. A. et al. Pictogram use was validated for estimating individual's body mass index. Journal of Clinical Epidemiology, v. 63, n. 6, p. 655-659, 2010.

KRUGER. J. et al. Body size satisfaction and physical activity levels among men and women. Obesity, v. 16, n. 8, p. 1976-1979, 2008.

KRUSTRUP, P.; BANGSBO, J. Physiological demands of top-class soccer refereeing in relation to physical capacity: effect of intense intermittent exercise training. Journal of Sports Science, v. 19, n. 11, p. 881-891, 2001.

LAGO-PEÑAS C.; GÓMEZ-LÓPEZ, M. The influence of referee bias on extra time in elite soccer matches. Perceptual and Motor Skills, v. 122, n. 2, p. 666-677, 2016

PANAYI, A. The Prevalence of Body Dysmorphic Disorder in Patients Undergoing Cosmetic Surgery: a Systematic Review. Psychiatria Danubina, v. 27, supl. 1, p. 438-444, set. 2015.

PAES, M. R.; FERNANDEZ, R.; DA SILVA, A. I. Injuries to football (soccer) referees during matches, training and physical tests. International SportMed Journal, v. 12, n. 2, p.74-84, 2011.

PHILLIPOU, A.; CASTLE, D. Body dysmorphic disorder in men. Australian Family Physician, v. 44, n. 11, p. 798801,2015

PIMENTA, A. M. et al. Relationship between body image disturbance and incidence of depression: The SUN prospective cohort. BMC Public Health, v. 9, s/n, p. 1, 2009 .
RONTOYANNIS, G. P. et al. Medical, morphological and functional aspects of Greek football referees. Journal of Sports Medicine and Physical Fitness. v. 38, n. 3, p. 208214, 1998.

STUNKARD, A. J.; SORENSON, T.; SCHLUSINGER, F. Use of the danish adoption register for the study of obesity and thinness. In: Kety, S. S. et al. The genetics of neurological and psychiatric disorders. New York: Raven, 1983. p. 115-120.

TYLKA, T. L.; WOOD-BARCALOW, N. L. What is and what is not positive body image? Conceptual foundations and construct definition. Body Image, v. 14, n. 4, p. 118$129,2015$.

ZORAGHI, M. R.; KHOSHNAM, E.; SOLHJO, M. H. Assessment of body composition in referees and assistant referees professional football. European Journal of Experimental Biology, v. 4, n. 1, p. 149-152, 2014

WILLIAMSON, D. A. et al. Body image assessment for obesity (BIA-O): development of a new procedure. International Journal of Obesity and Related Metabolic Disorders, v. 24, n. 10, p. 1326-1332, 2000.

WORLD HEALTH ORGANIZATION. Obesity: preventing and managing the global epidemic. World Health Organization: Geneva 1998. 276 p.

YEH, $\mathrm{H}$. The risk of eating disorders among female undergraduates in Taiwan. Archives of Psychiatric Nursing, v. 23 , n. 6, p. 430-440, 2009.

Recebido: 30/01/2018 Aceito: 15/07/2018 